\title{
Model Order Reduction for convection dominated problems
}

\section{H. Bansal ${ }^{1}$, L. lapichino ${ }^{1}$, S. Rave ${ }^{2}$, W.H.A. Schilders ${ }^{1}$, N. van de Wouw ${ }^{1,3,4}$ \\ ${ }^{1}$ Eindhoven University of Technology ${ }^{2}$ University of Muenster ${ }^{3}$ Delft University of Technology \\ ${ }^{4}$ University of Minnesota}

\section{Introduction}

Moving discontinuities are representative features of convection dominated problems and pose a major hindrance to obtain effective reduced-order model representations. An alternative Model Order Reduction (MOR) framework is proposed to efficiently capture such travelling discontinuities.

\section{Application Area}

Managed Pressure Drilling is used for drilling operations to guarantee the control of the down-hole pressure of the well (The schematic of the wellbore is shown in Figure 1 (left)). An accurate and simple enough hydraulic model needs to be employed in the context of real-time estimation and control in case of gas influx into the well.
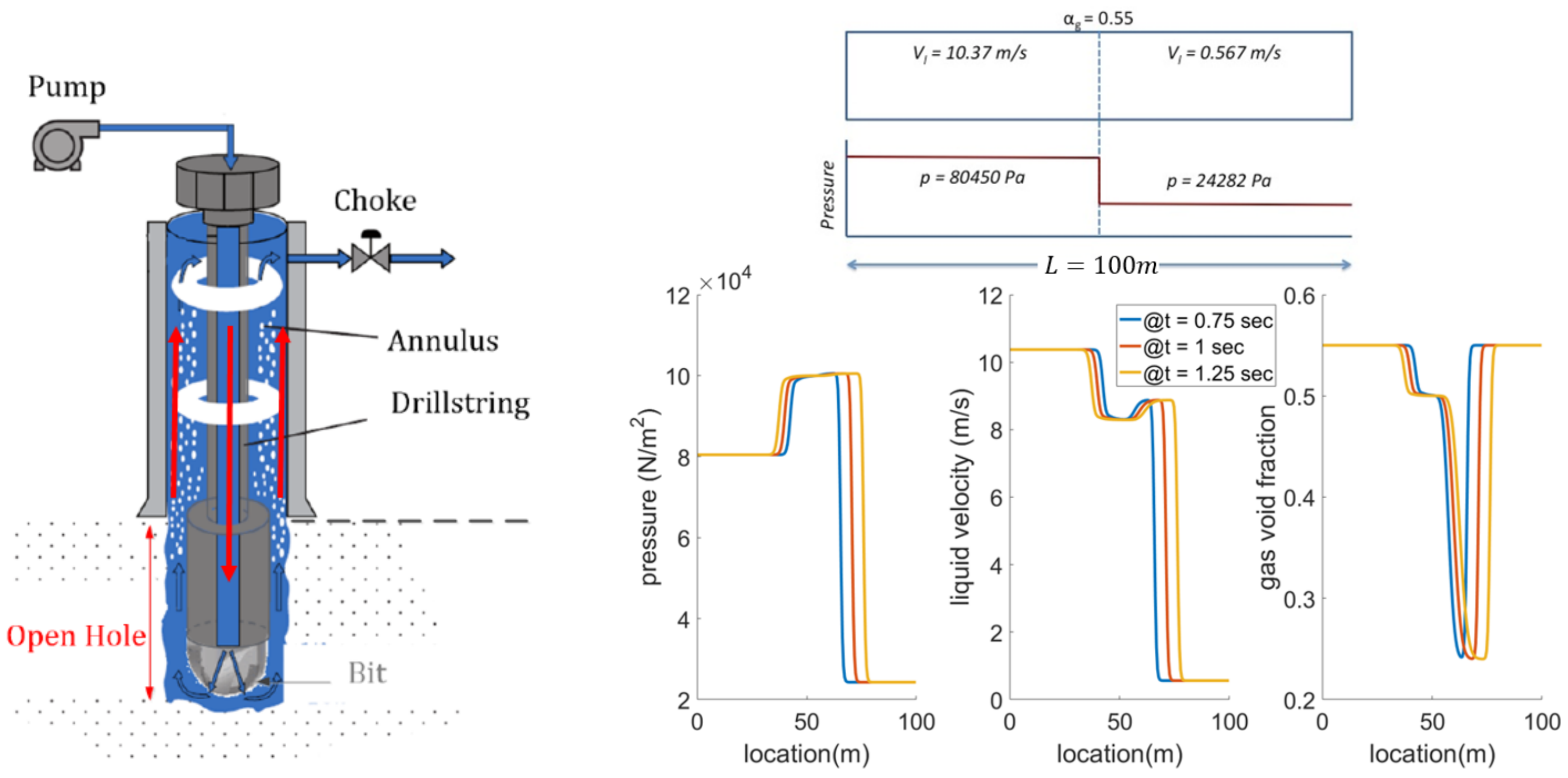

Figure 1: Wellbore Schematic (left) and Representative Solution of multi-phase shock tube test case (right).

The mathematical model, used to simulate the drilling operations, is called Drift Flux Model [2] It falls within the class of Hyperbolic Partial Differential Equations (PDEs). The representative features of this class of problems are represented in Figure 1 (right) for a specific multi-phase shock-tube test case. The model is highly complex and computationally expensive. Hence, a reduced-order model is essential.

\section{Concept, State of the Art and Prospective Areas of Improvement}

Key Idea: We factor out the travelling dynamics and build the MOR on the frozen dynamics.

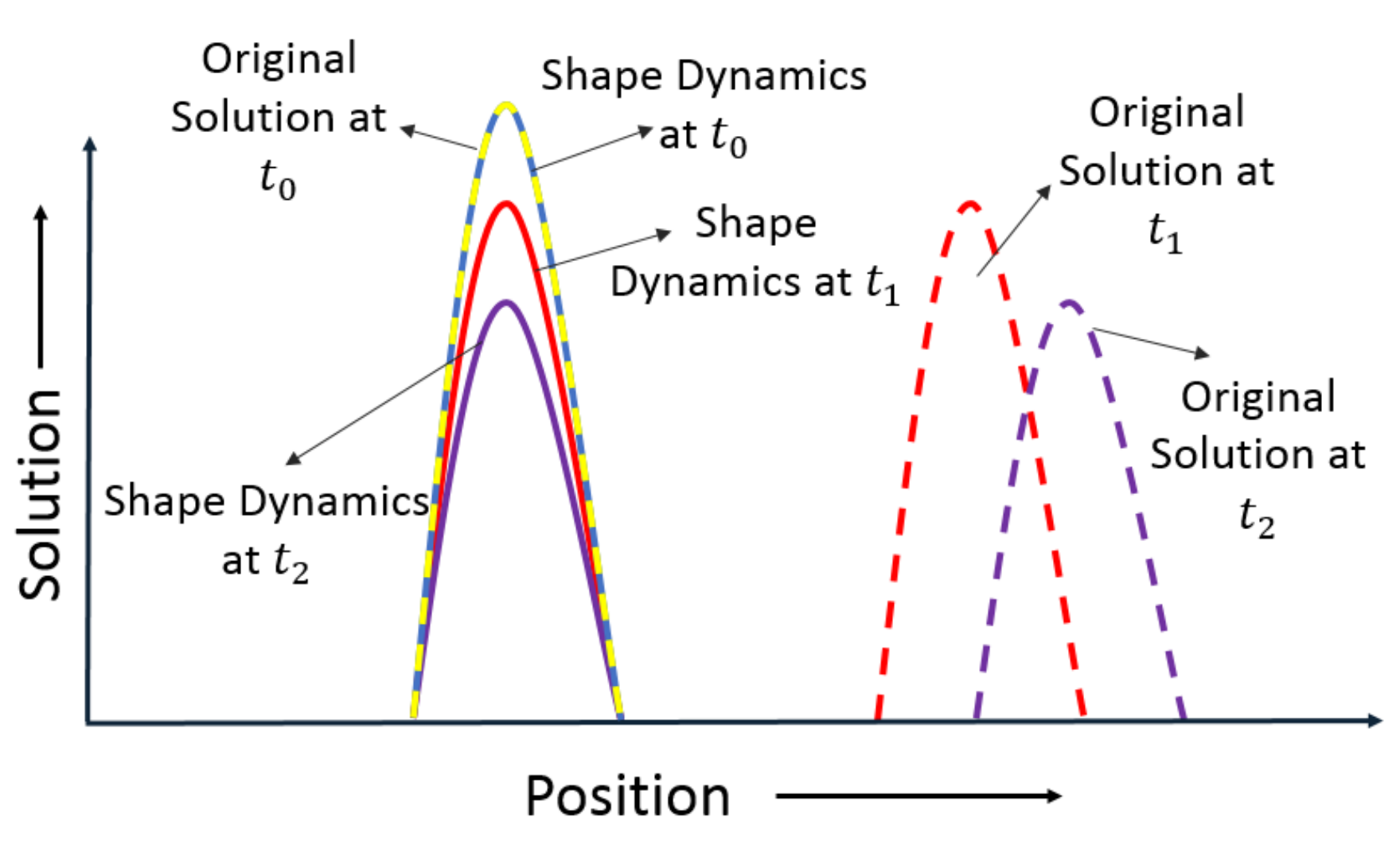

Figure 2: Concept Illustration: Here, Dot ted lines represent the evolution of original dynamics and solid lines represent the shape dynamics co-incide at $t_{0}=0$.

The concept of freezing multiple fronts/ pulses [1] has been applied for parabolic problems. However, such a concept has not been extended to hyperbolic problems. Moreover, the existing MOR framework [3], which builds on the Method of Freezing (for a single front), lacks consideration of boundary conditions and multiple fronts.

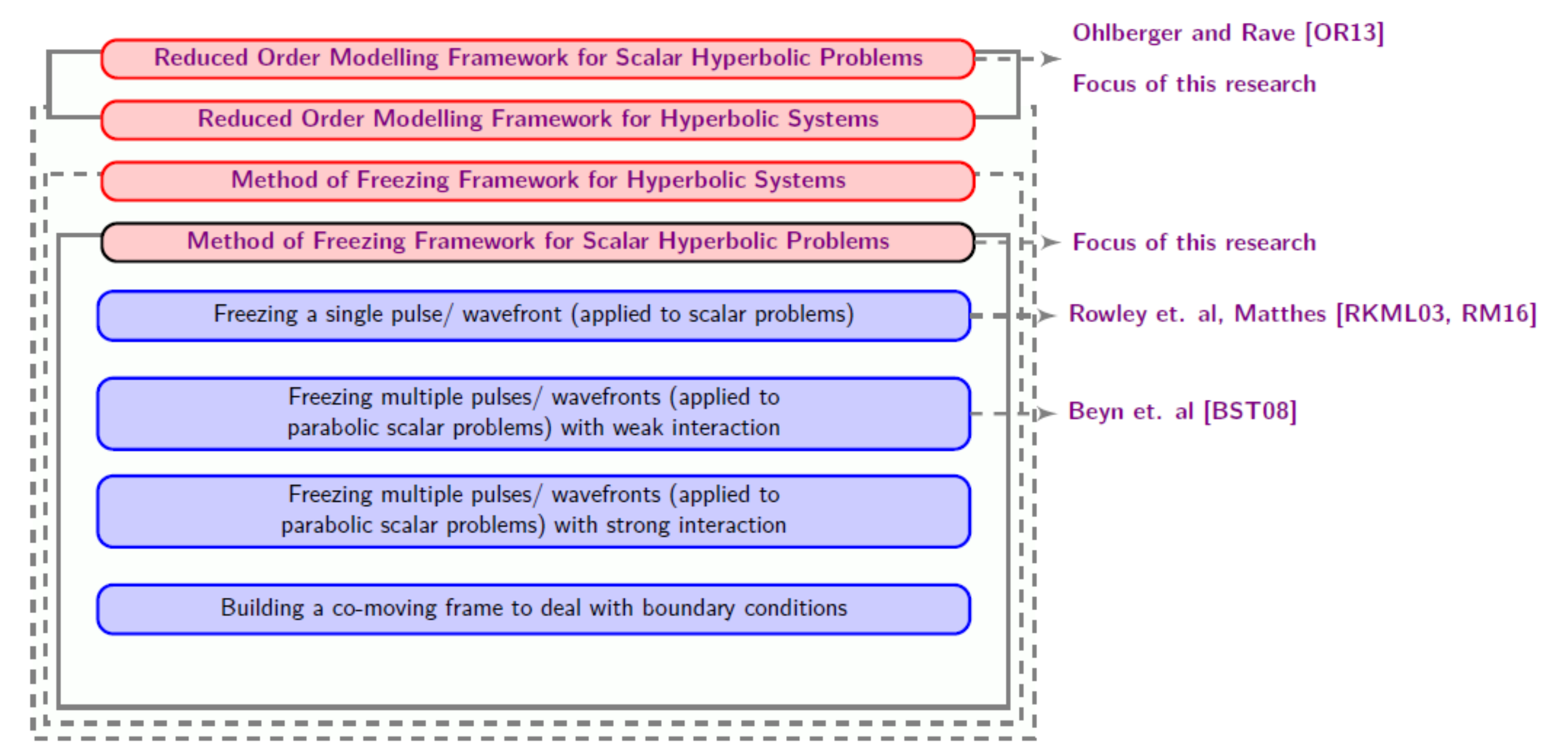

Figure 3: State of the Art, Prospective Areas of Improvement and Focus of this Research Figure 3 depicts the state of the art, prospective areas of improvement and the areas dealt with in this research. Our aim is to use underlying structure (wave propagation characteristics) to obtain smallest possible reduced-order model representation. We primarily investigate the performance of combined approach of Method of Freezing and reduced basis approximations in dealing with merging (discontinuous) wavefronts.

\section{Method of Freezing: Formulation}

Suppose, we have a governing PDE of the form: $\partial_{t} u=f\left(u, \partial_{x} u\right)$.

We construct a decomposition of the solution $u(x, t)$ in the following form:

$$
u(x, t)=\sum_{j=1}^{M} v_{j}\left(x-g_{j}(t), t\right) ; \xi_{j}=x-g_{j}(t) \text { and } M=\text { number of co-moving frames. }
$$

The group component $g_{j}(t)$ and shape dynamics $v_{j}\left(\xi_{j}, t\right) \in W_{N}$ (where $W_{N}$ is a "truth" solution space and $N$ is the dimension of the high fidelity model) are computed using Equations (1)-(3):

$$
\partial_{t} v_{j}\left(\xi_{j}, t\right)=\overbrace{\partial_{\xi_{j}} v_{j}\left(\xi_{j}, t\right)}^{H_{j, 1}} \mu_{j}(t)+\overbrace{\frac{\phi_{j}\left(\xi_{j}\right)}{\sum_{k=1}^{M} \phi_{k}\left(\xi_{k j}^{g}\right)}}^{Q_{j}^{g}\left(\xi_{j}, t\right)} \cdot \overbrace{f\left(\sum_{k=1}^{M} v_{k}\left(\xi_{k j}^{g}, t\right), \sum_{k=1}^{M} \partial_{\xi} v_{k}\left(\xi_{k j}^{g}, t\right)\right)}^{H_{j, j}^{\text {shifted }}} ; \xi_{k j}^{g}=\xi_{j}-g_{k}(t)+g_{j}(t),
$$

where $Q_{j}^{g}\left(\xi_{j}, t\right)$ form a time dependent partition of unity and $\mu_{j}$ is an additional degree of freedom, which is determined by imposing appropriate constraints:

$$
0=<\partial_{\xi_{j}} v_{j},(\underbrace{f\left(v_{j}, \partial_{\xi_{j}} v_{j}\right)}_{H_{j, 0}}+\mu_{j} \partial_{\xi_{j}} v_{j})>_{L^{2}},
$$

where, $\langle, .,\rangle_{L^{2}}$ is the inner-product. The rationale behind the above constraint is to force $v_{j}$ to have minimal change over time. The group component $g_{j}$ satisfies Equation (3).

$$
\partial_{t} g_{j}=\mu_{j}(t)
$$

5. Numerical Experiments (Freezing + MOR Framework) for Burgers Equation

Proper Orthogonal Decomposition (POD) framework: $v_{j, r}(., t) \in W_{r} \subset W_{N}$ for $t \in[0, T]$

$$
\begin{gathered}
\partial_{t} v_{j, r}=P_{V_{j, r}}\left[Q_{j}^{g} \cdot H_{j, 0}^{s h i f t e d}\right]+\left[\left(P_{V_{j, r}}\left[H_{j, 1}\right]\right) \cdot \mu_{j, r}\right] ; j=1,2, \ldots, M \\
{\left[H_{j, 1}\left(v_{j, r}\right)^{T} H_{j, 1}\left(v_{j, r}\right)\right] \cdot \mu_{j, r}=-\left[H_{j, 1}\left(v_{j, r}\right)^{T} H_{j, 0}\left(v_{j, r}\right)\right],} \\
\partial_{t} g_{j}=\mu_{j, r}(t) .
\end{gathered}
$$

where, $P_{V_{j .}}$ is projection basis, $r$ is the dimension of the reduced-order model and Equations (4)-(6) are the reduced analogues of Equations (1)-(3).

Remark 1 In the future, we will implement $P O D$ in conjunction with hyper-reduction techniques (such as Empirical Interpolation Method) to achieve cost savings and complexity reduction. Problem Setting: $u_{t}+u u_{x}=0$ under imposition of periodic boundary conditions. Figure 4 represents the initial settings of several numerical experiments.
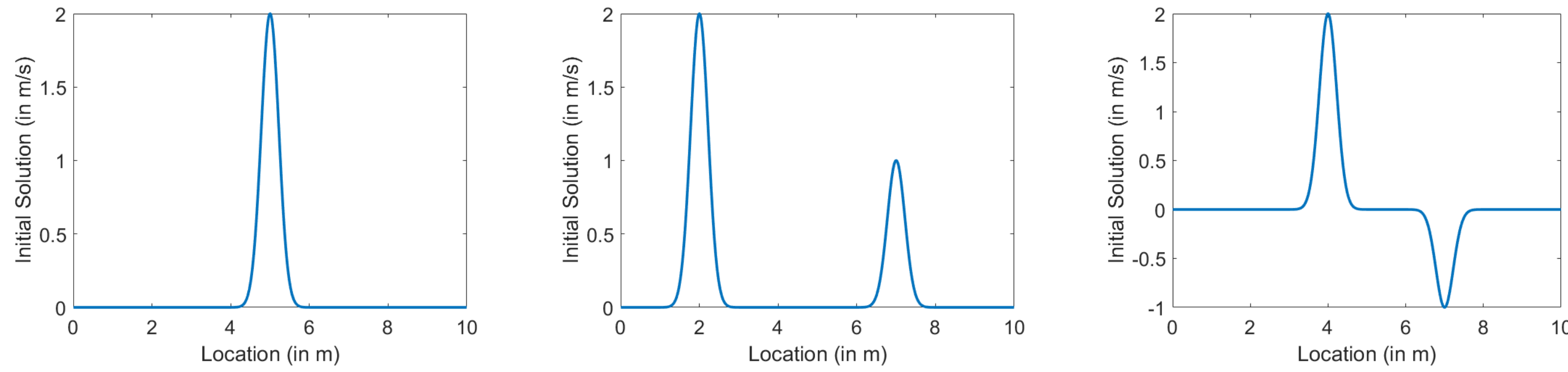

Figure 4: Initial conditions: Case 1 (left), Case 2a (middle) and Case 2b (right). - Case 1: Freezing a single front:

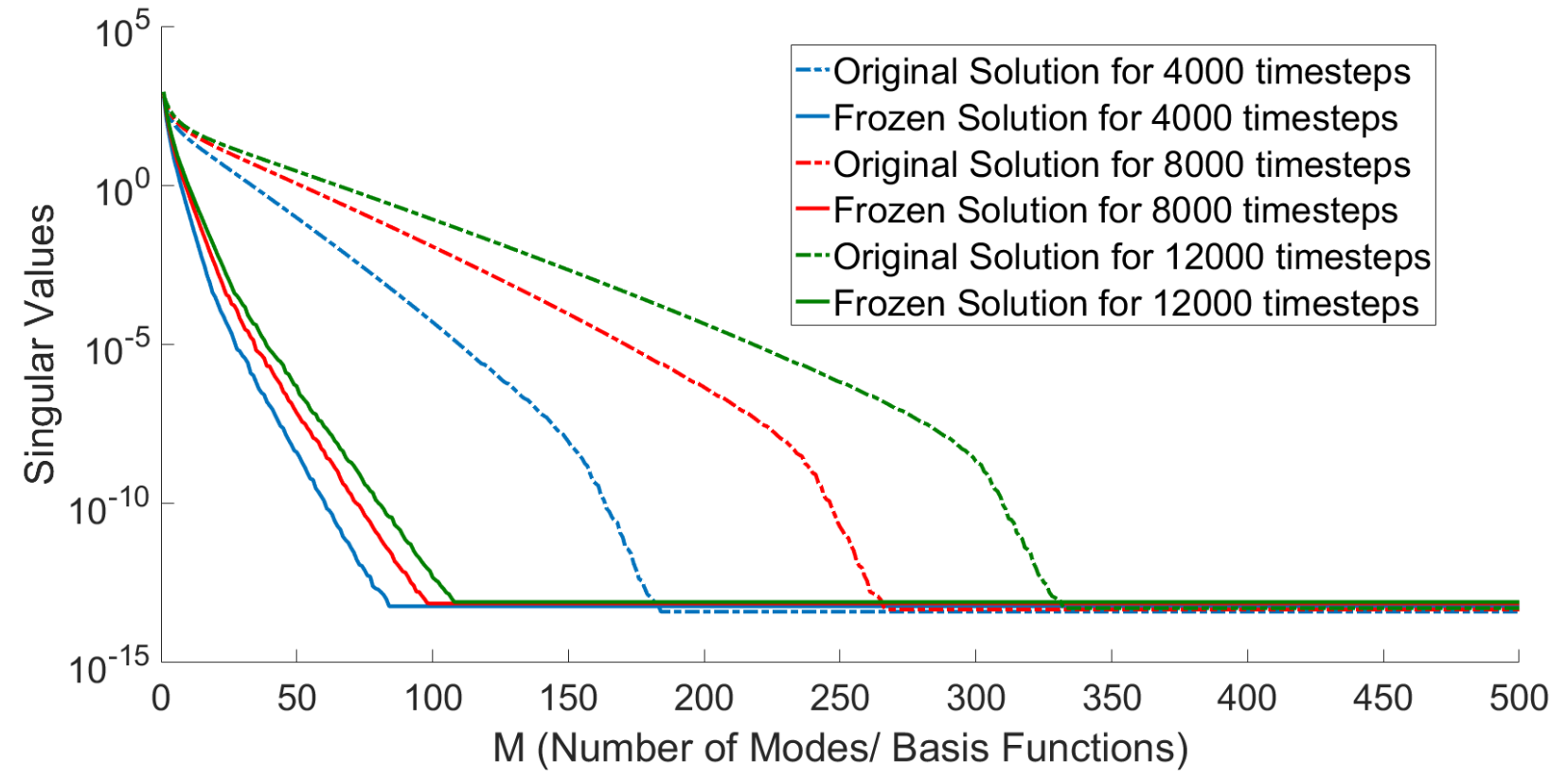

Figure 5: Singular Value Decay (for varying simulation runtimes) with and without freezing Figure 5 demonstrates that most of the basis are required to capture the travelling dynamics. Very few basis functions are required to capture the shape dynamics.

- Case 2: Freezing multiple fronts with: a) Weak Interaction and b) Strong Interaction

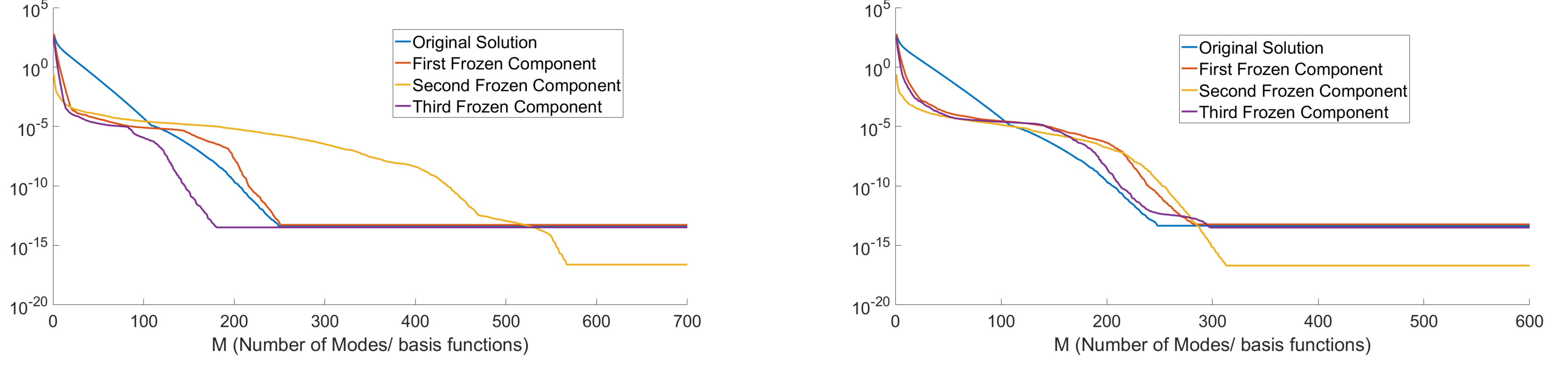

Figure 6: Singular Value Decay under weak interactions (left) and strong interactions (right) Figure 6 reveals that the frozen dynamics can be represented by a relatively small number of basis functions up to a certain level of solution accuracy. After a certain threshold accuracy level, shape dynamics require large number of basis functions for an efficient representation.

Representative numerical experiments can be accessed through scanning the QR code or visiting the website:

The numerical results encompass 1D scalar Burgers Equation.

\section{Conclusions and Future Plan}

Devising a MOR framework on top of method of freezing framework is promising. In the future we envision to:

- Extend this concept to handle stronger wave interactions/ non-periodic boundary conditions - Extend freezing framework to Euler Equations and Drift Flux Model

- Develop a reduced order model for multi-query simulations of drilling scenarios

\section{References}

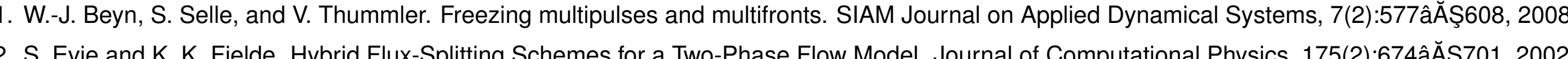

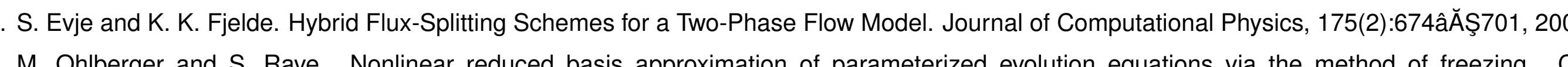

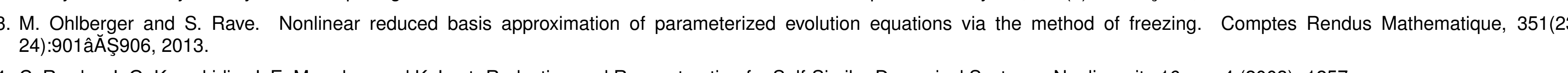

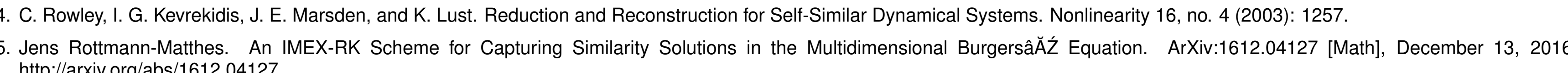

